\title{
The Fifth Dimension Subgroup for Metabelian 2 Groups
}

\author{
Shalini Gupta \\ Department of Mathematics, Punjabi University, Patiala 147002, India \\ Correspondence should be addressed to Shalini Gupta; gupta_math@yahoo.com
}

Received 18 April 2016; Accepted 14 June 2016

Academic Editor: Burkhard Külshammer

Copyright (c) 2016 Shalini Gupta. This is an open access article distributed under the Creative Commons Attribution License, which permits unrestricted use, distribution, and reproduction in any medium, provided the original work is properly cited.

Given a finite metabelian 2-group $G$, the object of this paper is to discuss some cases under which $D_{5}(G)=\gamma_{5}(G)$. Further, some examples of 2 groups of class 3 , for which $D_{4}(G) \neq\{1\}$ but $D_{5}(G)=\{1\}$, are discussed.

\section{Introduction}

Let $\mathbb{Z}(G)$ be the integral group ring of group $G$ and let $I(G)$ be the augmentation ideal of $\mathbb{Z}(G)$. For $n \geq 1$, let $D_{n}(G)=$ $G \cap\left\{1+I^{n}(G)\right\}$ be $n$th dimension subgroup and let $\gamma_{n}(G)$ be $n$th term in lower central series of group $G$. The famous dimension subgroup conjecture states that $D_{n}(G)=\gamma_{n}(G)$ for all $n \geq 1$ and for all groups $G$. This conjecture has been proved for $n \leq 3$ (see, e.g., [1]). It has been proved in [2] that if $G$ is a finite $p$-group, $p$ odd prime, then $D_{4}(G)=$ $\gamma_{4}(G)$. Keeping in view this result, Rips [3] gave an example of 2-group $G$ for which $D_{4}(G) \neq \gamma_{4}(G)$. Further Tahara [4] gave the structure of $D_{5}(G)$ and proved that the exponent of $D_{5}(G) / \gamma_{5}(G)$ is divisible by 6 . It has been shown in [5] that, for a metabelian group, the exponent of $D_{5}(G) / \gamma_{5}(G)$ is divisible by 2 , and hence for a metabelian $p$-group $G$, $p$ odd prime $D_{5}(G)=\gamma_{5}(G)$. Gupta [6] constructed a 2-group $G$, generated by four elements, such that $D_{5}(G) \neq \gamma_{5}(G)$. Now, the question arises that whether for a metabelian 2 -group $G$, with at most three generators, $D_{5}(G)=\gamma_{5}(G)$ or not.

In this paper, we prove that if $G$ is metabelian 2 group generated by at most two elements, then $D_{5}(G)=\gamma_{5}(G)$ (Theorem 2). Further we prove that under certain conditions, a metabelian 2-group $G$ generated by $n$ elements satisfies the dimension subgroup conjecture for $n=5$ (Theorem 3 ). Finally, in Section 3, we give some examples of 2 groups of class 3, for which $D_{5}(G)=\gamma_{5}(G)$.

\section{Main Results}

We first recall the structure of $D_{5}(G)$ given by Tahara [4]. Let $G$ be a finite group of class 4 . Consider the lower central series $G=\gamma_{1}(G) \supseteq \gamma_{2}(G) \supseteq \gamma_{3}(G) \supseteq \gamma_{4}(G) \supseteq \gamma_{5}(G)=\{1\}$ of $G$. Let $\left\{x_{1 i} \gamma_{2}(G)\right\}_{1 \leq i \leq s}$ be basis of $G / \gamma_{2}(G)$, with $d(i)$ as the order of $x_{1 i} \gamma_{2}(G)$ : that is, $x_{1 i}{ }^{d(i)}$ belongs to $\gamma_{2}(G)$. Similarly, let $\left\{x_{2 j} \gamma_{3}(G)\right\}_{1 \leq j \leq t}$ be basis of $\gamma_{2}(G) / \gamma_{3}(G)$, with $e(j)$ as the order of $x_{2 j} \gamma_{3}(G)$, and let $\left\{x_{3 k} \gamma_{4}(G)\right\}_{1 \leq k \leq u}$ be basis of $\gamma_{3}(G) / \gamma_{4}(G)$, with $f(k)$ as the order of $x_{3 k} \gamma_{4}(G)$. Moreover, these basis elements are chosen in such a way that $d(i)$ divides $d(i+1)$, $e(i)$ divides $e(i+1)$, and $f(i)$ divides $f(i+1)$. Thus, we have

$$
\begin{aligned}
& x_{1 i}{ }^{d(i)}=\prod_{1 \leq j \leq t} x_{2 j}{ }^{b_{i j}} \prod_{1 \leq k \leq u} x_{3 k}{ }^{c_{i k}} y_{4 i}, \\
& y_{4 i} \in \gamma_{4}(G), 1 \leq i \leq s ; \\
& x_{2 k}{ }^{e(k)}=\prod_{1 \leq k \leq u} x_{3 l}{ }^{d k l} y_{4 k}^{\prime}, \\
& {\left[x_{1 i}{ }^{d(i)}, x_{1 j}\right] }=\prod_{1 \leq l \leq u} x_{3 l}{ }^{\alpha^{(i j)}} y_{4 i j}^{\prime \prime}, \\
& y_{4 k}^{\prime} \in \gamma_{4}(G), 1 \leq k \leq t ; \\
& y_{4 i j}^{\prime \prime} \in \gamma_{4}(G), 1 \leq i \leq s .
\end{aligned}
$$

The following theorem gives the structure of $D_{5}(G)$. 
Theorem 1 (see [4]). With the above notations, $D_{5}(G)$ is equal to the subgroup generated by the elements

$$
\begin{aligned}
& \prod_{1 \leq i<j \leq s}\left[x_{1 i}{ }^{d(i)}, x_{1 j}\right]^{u_{i j}(d(j) / d(i))} \prod_{1 \leq i \leq s, 1 \leq k \leq t} \prod_{k<l}\left[x_{2 l}, x_{2 k}\right]^{b_{i l} v_{i k}} \\
& \cdot \prod_{1 \leq i \leq j \leq k \leq s}\left[x_{1 i}^{d(i)}, x_{1 j}, x_{1 k}\right]^{w_{i j k}}
\end{aligned}
$$

with the following conditions:

$$
\begin{aligned}
& w_{i i i}=0, \quad 1 \leq i \leq s ; \\
& u_{i j} \frac{d(j)}{d(i)}\left(\begin{array}{c}
d(i) \\
2
\end{array}\right)+w_{i i j} d(i)+w_{i i j}^{\prime \prime} d(j)=0, \\
& \qquad 1 \leq i<j \leq s ; \\
& -u_{i j}\left(\begin{array}{c}
d(j) \\
2
\end{array}\right)+w_{i j j} d(i)+w_{i j j}^{\prime} d(j)=0,
\end{aligned}
$$$$
1 \leq i<j \leq s
$$$$
w_{i j k} d(i)+w_{i j k}^{\prime} d(j)+w_{i j k}^{\prime \prime} d(k)=0,
$$$$
1 \leq i<j<k \leq s
$$$$
\sum_{i<h} u_{i h} b_{h k}-\sum_{h<i} u_{h i} \frac{d(i)}{d(h)} b_{h k}+v_{i k} d(i)+v_{i k}^{\prime} e(k)=0,
$$$$
1 \leq i \leq s, 1 \leq k \leq t
$$$$
u_{i j} \frac{d(j)}{d(i)}\left(\begin{array}{c}
d(i) \\
3
\end{array}\right)+w_{i i j}\left(\begin{array}{c}
d(i) \\
2
\end{array}\right) \equiv 0
$$

$(\bmod d(i)), 1 \leq i<j \leq s ;$

$$
w_{i i j}\left(\begin{array}{c}
d(i) \\
2
\end{array}\right)+w_{i i j}^{\prime \prime}\left(\begin{array}{c}
d(j) \\
2
\end{array}\right) \equiv 0
$$

$(\bmod d(i)), 1 \leq i<j \leq s ;$

$$
-u_{i j}\left(\begin{array}{c}
d(j) \\
3
\end{array}\right)+w_{i j j}^{\prime}\left(\begin{array}{c}
d(j) \\
2
\end{array}\right) \equiv 0
$$

$(\bmod d(i)), 1 \leq i<j \leq s ;$

$$
w_{i j k}\left(\begin{array}{c}
d(i) \\
2
\end{array}\right), w_{i j k}^{\prime}\left(\begin{array}{c}
d(j) \\
2
\end{array}\right), w_{i j k}^{\prime \prime}\left(\begin{array}{c}
d(k) \\
2
\end{array}\right) \equiv 0
$$

$(\bmod d(i)), 1 \leq i<j<k \leq s ;$

$$
\begin{aligned}
& v_{i k}\left(\begin{array}{c}
d(i) \\
2
\end{array}\right)- \sum_{h \leq i} w_{h i i} b_{h k}-\sum_{i<h} w_{i i h}^{\prime \prime} b_{h k} \equiv 0 \\
&(\bmod (d(i), e(k))), 1 \leq i \leq s, 1 \leq k \leq t ; \\
& \sum_{h \leq i} w_{h i j} b_{h k}+\sum_{i<h \leq j} w_{i h j}^{\prime} b_{h k}+\sum_{j<h} w_{i j k}^{\prime \prime} b_{h k} \equiv 0 \\
&(\bmod (d(i), f(l))), 1 \leq i \leq s, 1 \leq l \leq u ;
\end{aligned}
$$

Thus,

$$
\begin{aligned}
& -\sum_{h<i} u_{h i} \frac{d(i)}{d(h)} \alpha_{l}^{(h i)}+\sum_{i<h} u_{i h} c_{h i}-\sum_{h<i} u_{h i} \frac{d(i)}{d(h)} c_{h i} \\
& -\sum_{k} v_{i k}^{\prime} d_{k l}-\sum_{g \leq i \leq h} w_{g i h} \alpha_{l}^{(g h)}-\sum_{g \leq h \leq i} w_{g h i} \alpha_{l}^{(g h)} \\
& -\sum_{i<g \leq h} w_{i g h}^{\prime} \alpha_{l}^{(g h)} \equiv 0
\end{aligned}
$$

$$
(\bmod (d(i), f(l))), 1 \leq i \leq s, 1 \leq l \leq u \text {; }
$$

$$
\begin{aligned}
& \sum_{i} v_{i k} b_{i k} \equiv 0 \quad(\bmod e(k)), 1 \leqslant k \leqslant t ; \\
& \sum_{i} v_{i k} b_{i l}+\sum_{i} v_{i l} b_{i k} \equiv 0 \quad(\bmod e(k)), 1 \leqslant k<l \leqslant t .
\end{aligned}
$$

Theorem 2. Let $G$ be metabelian 2 group with $G / \gamma_{2}(G)$ as sum of at most two cyclic groups. Then $D_{5}(G)=\gamma_{5}(G)$.

Proof. It is obvious that if metabelian 2 groups are such that $G / \gamma_{2}(G)$ is a cyclic group then $D_{5}(G)=\gamma_{5}(G)$.

Let $G$ be metabelian 2 group and let $G / \gamma_{2}(G)$ be sum of two cyclic groups. It is enough to prove the result for a group of class 4 . Let $G / \gamma_{2}(G)=C_{1} \oplus C_{2}$, where $C_{i}$ is a cyclic group generated by $x_{1 i} \gamma_{2}(G)$ with order of $x_{1 i} \gamma_{2}(G)=d(i)$ and $d(1)$ divides $d(2)$. An arbitrary element $g$ of $D_{5}(G)$ is of the form $\left[x_{11}{ }^{d(1)}, x_{12}\right]^{u_{12}(d(2) / d(1))}\left[x_{11}{ }^{d(1)}, x_{12}, x_{12}\right]^{w_{122}}$ subject to the conditions given in Theorem 1 . For $1 \leq k \leq t$ and $i=2$, (9) becomes

$$
-u_{12} \frac{d(2)}{d(1)} b_{1 k}+v_{2 k} d(2)+v_{2 k}^{\prime} e(k)=0 .
$$

$$
\begin{aligned}
& 1=\prod_{k=1}^{t}\left[x_{2 k}, x_{12}\right]^{-u_{12}(d(2) / d(1)) b_{1 k}+v_{2 k} d(2)+v_{2 k}^{\prime} e(k)} \\
& =\prod_{k=1}^{t}\left\{\left[x_{2 k}{ }^{b_{1 k}}, x_{12}\right]^{-u_{12}(d(2) / d(1))}\right. \\
& \left.\cdot\left[x_{2 k}, x_{12}, x_{2 k}\right]^{u_{12}(d(2) / d(1))\left(\begin{array}{c}
b_{1 k} \\
2
\end{array}\right)}\right\}
\end{aligned}
$$

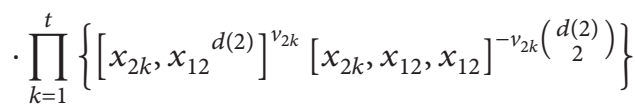

$$
\begin{aligned}
& \left.\cdot \prod_{k=1}^{t}\left\{\left[x_{2 k}^{e(k)}, x_{12}\right]^{v_{2 k}^{\prime}}\left[x_{2 k}, x_{12}, x_{2 k}\right]^{-v_{2 k}^{\prime}(e(k)} \begin{array}{c}
(e) \\
2
\end{array}\right)\right\} .
\end{aligned}
$$

Now $\left[x_{2 k}, x_{12}, x_{2 k}\right]$ belongs to $\gamma_{5}(G)$ and $\left[x_{2 k}, x_{12}{ }^{d(2)}\right]$ belongs to $\left[\gamma_{2}(G), \gamma_{2}(G)\right]$; thus we have

$$
\begin{aligned}
1= & \prod_{k=1}^{t}\left[x_{2 k} b_{1 k}, x_{12}\right]^{-u_{12}(d(2) / d(1))} \\
& \cdot \prod_{k=1}^{t}\left[x_{2 k}, x_{12}, x_{12}\right]^{-v_{2 k}\left(\frac{d(2)}{2}\right)} \prod_{k=1}^{t}\left[x_{2 k}^{e(k)}, x_{12}\right]^{v_{2 k}^{\prime}}
\end{aligned}
$$




$$
\begin{aligned}
& =\left[\prod_{k=1}^{t} x_{2 k} b_{1 k}, x_{12}\right]^{-u_{12}(d(2) / d(1))} \\
& \cdot \prod_{k=1}^{t}\left[x_{2 k}, x_{12}, x_{12}\right]^{-v_{2 k} d\left(\begin{array}{c}
d(2) \\
2
\end{array}\right)} \prod_{k=1}^{t} \prod_{l=1}^{u}\left[x_{3 l}^{d_{k l}}, x_{12}\right]^{v_{2 k}^{\prime}} \\
& =\left[\prod_{k=1}^{t} x_{2 k} b_{1 k}, x_{12}\right]^{-u_{12}(d(2) / d(1))} \\
& \cdot \prod_{k=1}^{t}\left[x_{2 k}, x_{12}, x_{12}\right]^{-w_{122} b_{1 k}} \prod_{l=1}^{u}\left[x_{3 l}, x_{12}\right]^{\sum_{k} v_{2 k}^{\prime} d_{k l}},
\end{aligned}
$$

as for $i=2(14)$ becomes $v_{2 k}\left(\begin{array}{c}d(2) \\ 2\end{array}\right)-w_{122} b_{1 k} \equiv 0(\bmod (d(2)$, $e(k)))$. Now, (16), for $i=2$, becomes

$$
\begin{gathered}
-u_{12} \frac{d(2)}{d(1)} \alpha_{l}^{(12)}-u_{12} \frac{d(2)}{d(1)} c_{1 l}-\sum_{k} v_{2 k}^{\prime} d_{k l}-2 w_{122} \alpha_{l}^{(12)} \\
-w_{112} \alpha_{l}^{(11)} \equiv 0 \quad(\bmod (d(2), f(l)))
\end{gathered}
$$

which implies that

$$
\begin{aligned}
& \prod_{l=1}^{u}\left[x_{3 l}, x_{12}\right]^{\sum_{k} v_{2 k}^{\prime} d_{k l}}=\prod_{l=1}^{u}\left[x_{3 l},\right. \\
& \left.x_{12}\right]^{-u_{12}(d(2) / d(1)) \alpha_{l}^{(12)}-u_{12}(d(2) / d(1)) c_{1 l}-2 w_{122} \alpha_{l}^{(12)}-w_{112} \alpha_{l}^{(11)}} \\
& =\left[\prod_{l=1}^{u} x_{3 l}^{c_{1 l}}, x_{12}\right]^{-u_{12}(d(2) / d(1))}\left[\prod_{l=1}^{u} x_{3 l} \alpha_{l}^{(12)},\right. \\
& \left.x_{12}\right]^{-u_{12}(d(2) / d(1))}\left[\prod_{l=1}^{u} x_{3 l} \alpha_{l}^{(12)}, x_{12}\right]^{-2 w_{122}}\left[\prod_{l=1}^{u} x_{3 l} \alpha_{l}^{(11)},\right. \\
& \left.x_{12}\right]^{-w_{112}}=\left[\prod_{l=1}^{u} x_{3 l}^{c_{1 l}, x_{12}}\right]^{-u_{12}(d(2) / d(1))}\left[x_{11}^{d(1)},\right. \\
& \left.x_{12}, x_{12}\right]^{-u_{12}(d(2) / d(1))}\left[x_{11} d(1), x_{12}, x_{12}\right]^{-2 w_{122}} .
\end{aligned}
$$

Now,

$$
\begin{aligned}
& {\left[x_{11}^{d(1)}, x_{12}, x_{12}\right]^{-u_{12}(d(2) / d(1))}=\left[x_{11}, x_{12}, x_{12}\right]^{-u_{12} d(2)}} \\
& \cdot\left[x_{12}, x_{11}, x_{11}, x_{12}\right]^{u_{12}(d(2) / d(1))\left(\begin{array}{c}
d(1) \\
2
\end{array}\right)} \\
& =\left[x_{11}, x_{12}, x_{12}^{d(2)}\right]^{-u_{12}} \\
& \cdot\left[x_{12}, x_{11}, x_{12}, x_{12}\right]^{-u_{12}\left(\begin{array}{c}
d(2) \\
2
\end{array}\right)} \\
& \cdot\left[x_{12}, x_{11}, x_{11}, x_{12}\right]^{u_{12}(d(2) / d(1))\left(\begin{array}{c}
d(1) \\
2
\end{array}\right)} .
\end{aligned}
$$

Using (6) and (7), we get that

$$
\begin{aligned}
u_{12} \frac{d(2)}{d(1)}\left(\begin{array}{c}
d(1) \\
2
\end{array}\right) \equiv 0 \quad(\bmod d(1)) \\
-u_{12}\left(\begin{array}{c}
d(2) \\
2
\end{array}\right) \equiv 0 \quad(\bmod d(1)) .
\end{aligned}
$$

Hence, (24) reduces to

$$
\begin{gathered}
{\left[x_{11}^{d(1)}, x_{12}, x_{12}\right]^{-u_{12}(d(2) / d(1))}=\left[x_{11}, x_{12}, x_{12}^{d(2)}\right]^{-u_{12}}} \\
=1, \quad \text { as }\left[x_{11}, x_{12}, x_{12}^{d(2)}\right] \in\left[\gamma_{2}(G), \gamma_{2}(G)\right] .
\end{gathered}
$$

Now,

$$
\begin{aligned}
& {\left[x_{11}^{d(1)}, x_{12}, x_{12}\right]^{-2 w_{122}}=\left[x_{11}, x_{12}, x_{12}\right]^{-2 w_{122} d(1)}} \\
& \cdot\left[x_{12}, x_{11}, x_{11}, x_{12}\right]^{2 w_{122}\left(\begin{array}{c}
d(1) \\
2
\end{array}\right)} \\
& \quad=\left[x_{11}, x_{12}, x_{12}\right]^{-2 w_{122} d(1)} .
\end{aligned}
$$

Also (7) implies that

$$
-2 w_{122} d(1)=-2 u_{12}\left(\begin{array}{c}
d(2) \\
2
\end{array}\right)+2 w_{122}^{\prime} d(2) .
$$

Thus, (28) becomes

$$
\begin{aligned}
& {\left[x_{11}^{d(1)}, x_{12}, x_{12}\right]^{-2 w_{122}}=\left[x_{11}, x_{12}, x_{12}\right]^{2 w_{122}^{\prime} d(2)}} \\
& \cdot\left[x_{11}, x_{12}, x_{12}\right]^{-u_{12} d(2)(d(2)-1)} \\
& \quad=\left[x_{11}, x_{12}, x_{12}^{d(2)}\right]^{2 w_{122}^{\prime}} \\
& \cdot\left[x_{12}, x_{11}, x_{12}, x_{12}\right]^{-2 w_{122}^{\prime}\left(\begin{array}{c}
d(2) \\
2
\end{array}\right)} \\
& \cdot\left[x_{11}, x_{12}, x_{12}\right]^{-u_{12}(d(2)(d(2)-1))} \\
& =\left[x_{11}, x_{12}, x_{12}^{d(2)}\right]^{-u_{12}(d(2)-1)} \\
& \cdot\left[x_{12}, x_{11}, x_{12}, x_{12}\right]^{-u_{12}\left((d(2)-1)\left(\begin{array}{c}
d(2) \\
2
\end{array}\right)\right)}=1,
\end{aligned}
$$

as by $(7),-u_{12}\left(\begin{array}{c}d(2) \\ 2\end{array}\right) \equiv 0(\bmod d(1))$.

Using (23), (27), and (30) in (21), we get that

$$
\begin{aligned}
1 & =\left[\prod_{k=1}^{t} x_{2 k}{ }^{b_{1 k}}, x_{12}\right]^{-u_{12}(d(2) / d(1))} \\
& \cdot\left[\prod_{l=1}^{u} x_{3 l}^{c_{1 l}}, x_{12}\right]^{-u_{12}(d(2) / d(1))} \\
& \cdot\left[\prod_{k=1}^{t} x_{2 k}{ }^{b_{1 k}}, x_{12}, x_{12}\right]^{-w_{122}} \\
& =\left[x_{11}^{d(1)}, x_{12}\right]^{-u_{12}(d(2) / d(1))}\left[x_{11}^{d(1)}, x_{12}, x_{12}\right]^{-w_{122}} \\
& =g^{-1} .
\end{aligned}
$$

Thus, $g=1$, and hence $D_{5}(G)=\{1\}$. 
Theorem 3. Let $G$ be finite metabelian 2 group and $G / \gamma_{2}(G)=$ $C_{1} \oplus C_{2} \oplus \cdots \oplus C_{n}$, where $C_{i}$ are cyclic groups of order $d(i)$. Let $d(1)=d(2)=\cdots=d(n-1)=2, d(n) \geq 4$. Then $D_{5}(G)=$ $\gamma_{5}(G)$.

Proof. It is enough to prove the result for a group $G$ of class 4 . Any element $g$ of $D_{5}(G)$ is of the form

$$
\begin{aligned}
g= & \prod_{1 \leq i<j \leq n}\left[x_{1 i}^{d(i)}, x_{1 j}\right]^{u_{i j}(d(j) / d(i))} . \\
& \prod_{1 \leq i<j<k \leq n}\left[x_{1 i}^{d(i)}, x_{1 j}, x_{1 k}\right]^{w_{i j k}} . \\
& \prod_{1 \leq i<j \leq n}\left[x_{1 i}^{d(i)}, x_{1 j}, x_{1 j}\right]^{w_{i j j}}=A \cdot B \cdot C \text { say. }
\end{aligned}
$$

Since for $1 \leq i<j<k \leq n, d(i)=d(j)=2$, (13) reduces to

$$
w_{i j k} \equiv 0 \quad(\bmod d(j))
$$

and hence $B=1$. Now,

$$
\begin{aligned}
A & =\prod_{1 \leq i<j \leq n-1}\left[x_{1 i}{ }^{d(i)}, x_{1 j}\right]^{u_{i j}(d(j) / d(i))} \\
& \cdot \prod_{i=1}^{n-1}\left[x_{1 i}^{d(i)}, x_{1 n}\right]^{u_{i n}(d(n) / d(i))} \\
& =\prod_{1 \leq i<j \leq n-1}\left[x_{1 i}{ }^{d(i)}, x_{1 j}\right]^{u_{i j}} \\
& \cdot \prod_{i=1}^{n-1}\left[x_{1 i}^{d(i)}, x_{1 n}\right]^{u_{i n}(d(n) / d(i))}
\end{aligned}
$$$$
\text { as for } 1 \leq i<j \leq n-1, d(i)=d(j) \text {. }
$$

Also for $1 \leq i<j \leq n$, (7) implies that $-u_{i j}+2 w_{i j j}+2 w_{i j j}^{\prime}=0$, which gives that

$$
\begin{aligned}
& \prod_{1 \leq i<j \leq n-1}\left[x_{1 i}{ }^{d(i)}, x_{1 j}\right]^{u_{i j}} \\
= & \prod_{1 \leq i<j \leq n-1}\left[x_{1 i}{ }^{d(i)}, x_{1 j}\right]^{2 w_{i j j}+2 w_{i j j}^{\prime}}, \text { as } d(j)=2 \\
= & \prod_{1 \leq i<j \leq n-1}\left[x_{1 i}^{d(i)}, x_{1 j}\right]^{d(j)\left(w_{i j j}+w_{i j j}^{\prime}\right)} \\
= & \prod_{1 \leq i<j \leq n-1}\left\{\left[x_{1 i}{ }^{d(i)}, x_{1 j}^{d(j)}\right]^{\left(w_{i j j}+w_{i j j}^{\prime}\right)}\right.
\end{aligned}
$$

$$
\begin{aligned}
& \left.\cdot\left[x_{1 i}^{d(i)}, x_{1 j}, x_{1 j}\right]^{-\left(w_{i j j}+w_{i j j}^{\prime}\right)\left(\begin{array}{c}
d(j) \\
2
\end{array}\right)}\right\} \\
& =\prod_{1 \leq i<j \leq n-1}\left[x_{1 i}^{d(i)}, x_{1 j}, x_{1 j}\right]^{-w_{i j j}} \\
& \cdot \prod_{1 \leq i<j \leq n-1}\left[x_{1 i}^{d(i)}, x_{1 j}, x_{1 j}\right]^{-w_{i j j}^{\prime}} \\
& \text { as }\left[x_{1 i}{ }^{d(i)}, x_{1 j}{ }^{d(j)}\right] \in\left[\gamma_{2}(G), \gamma_{2}(G)\right], d(j)=2 .
\end{aligned}
$$

Now for $1 \leq i<j \leq n$, (12) implies that $w_{i j j}^{\prime} \equiv 0(\bmod d(j))$, which gives that

$$
\begin{aligned}
& \prod_{1 \leq i<j \leq n-1}\left[x_{1 i}^{d(i)}, x_{1 j}\right]^{u_{i j}} \\
& =\prod_{1 \leq i<j \leq n-1}\left[x_{1 i}^{d(i)}, x_{1 j}, x_{1 j}\right]^{-w_{i j j}} .
\end{aligned}
$$

Thus, (34) reduces to

$$
\begin{aligned}
A= & \prod_{i=1}^{n-1}\left[x_{1 i}^{d(i)}, x_{1 n}\right]^{u_{i n}(d(n) / d(i))} \\
& \cdot \prod_{1 \leq i<j \leq n-1}\left[x_{1 i}{ }^{d(i)}, x_{1 j}, x_{1 j}\right]^{-w_{i j j}} .
\end{aligned}
$$

Hence,

$$
\begin{aligned}
g= & \prod_{i=1}^{n-1}\left[x_{1 i}^{d(i)}, x_{1 n}\right]^{u_{i n}(d(n) / d(i))} \\
& \cdot \prod_{i=1}^{n-1}\left[x_{1 i}^{d(i)}, x_{1 n}, x_{1 n}\right]^{w_{i n n}} .
\end{aligned}
$$

Now, for $i=n$, (9) becomes

$$
S=-\sum_{j=1}^{n-1} u_{j n} \frac{d(n)}{d(j)} b_{j k}+v_{n k} d(n)+v_{n k}^{\prime} e(k)=0,
$$

which gives

$$
\begin{aligned}
& 1=\prod_{k=1}^{t}\left[x_{2 k}, x_{1 n}\right]^{S}=\prod_{k=1}^{t}\left[x_{2 k}, x_{1 n}\right]^{-\sum_{j=1}^{n-1} u_{j n}(d(n) / d(j)) b_{j k}} \\
& \cdot \prod_{k=1}^{t}\left[x_{2 k}, x_{1 n}\right]^{v_{n k} d(n)} \prod_{k=1}^{t}\left[x_{2 k}, x_{1 n}\right]^{v_{n k}^{\prime} e(k)} \\
& =\prod_{k=1}^{t}\left[\prod_{j=1}^{n-1} x_{2 k}^{b_{j k}}, x_{1 n}\right]^{-u_{j n}(d(n) / d(j))} \\
& \cdot \prod_{k=1}^{t}\left\{\left[x_{2 k}, x_{1 n}^{d(n)}\right]^{v_{n k}}\left[x_{2 k}, x_{1 n}, x_{1 n}\right]^{-v_{n k}\left(\begin{array}{c}
d(n) \\
2
\end{array}\right)}\right\} \\
& \cdot \prod_{k=1}^{t}\left[x_{2 k}^{e(k)}, x_{1 n}\right]^{v_{n k}^{\prime}}
\end{aligned}
$$




$$
\begin{aligned}
& =\prod_{k=1}^{t}\left[\prod_{j=1}^{n-1} x_{2 k}^{b_{j k}}, x_{1 n}\right]^{-u_{j n}(d(n) / d(j))} \\
& \cdot \prod_{k=1}^{t}\left[x_{2 k}, x_{1 n}, x_{1 n}\right]^{-v_{n k}\left(\begin{array}{c}
d(n) \\
2
\end{array}\right)} \prod_{k=1}^{t} \prod_{l=1}^{u}\left[x_{3 l} d_{k l}, x_{1 n}\right]^{v_{n k}^{\prime}} \\
& =\prod_{k=1}^{t}\left[\prod_{j=1}^{n-1} x_{2 k} b_{j k}, x_{1 n}\right]^{-u_{j n}(d(n) / d(j))} \\
& \cdot \prod_{k=1}^{t}\left[x_{2 k}, x_{1 n}, x_{1 n}\right]^{-v_{n k}\left(\begin{array}{c}
d(n) \\
2
\end{array}\right)} \prod_{l=1}^{u}\left[x_{3 l}, x_{1 n}\right]^{\sum_{k} v_{n k}^{\prime} d_{k l}} .
\end{aligned}
$$

Equation (14) for $i=n$ becomes

$$
v_{n k}\left(\begin{array}{c}
d(n) \\
2
\end{array}\right) \equiv \sum_{j=1}^{n-1} w_{j n n} b_{j k} \quad(\bmod (d(n), e(k))) .
$$

Thus,

$$
\begin{aligned}
\prod_{k=1}^{t} & {\left[x_{2 k}, x_{1 n}, x_{1 n}\right]^{-v_{n k}\left(\begin{array}{c}
d(n) \\
2
\end{array}\right)} } \\
& =\prod_{i=1}^{n-1}\left[\prod_{k} x_{2 k}{ }^{b_{j k}}, x_{1 n}, x_{1 n}\right]^{-w_{i n n}} \\
& =\prod_{i=1}^{n-1}\left[x_{1 i}{ }^{d(i)}, x_{1 n}, x_{1 n}\right]^{-w_{i n n}}
\end{aligned}
$$

Using (16), we get that

$$
\begin{aligned}
\prod_{l=1}^{u} & {\left[x_{3 l}, x_{1 n}\right]^{\sum_{k} v_{n k}^{\prime} d_{k l}} } \\
& =\prod_{i=1}^{n-1}\left[x_{1 i}^{d(i)}, x_{1 n}, x_{1 n}\right]^{-u_{i n}(d(n) / d(i))} \\
& \cdot \prod_{i=1}^{n-1}\left[x_{1 i}^{d(i)}, x_{1 n}, x_{1 n}\right]^{-2 w_{i n n}} \\
& \cdot \prod_{i=1}^{n-1}\left[\prod_{l} x_{3 l}^{c_{i l}}, x_{1 n}\right]^{-u_{i n}(d(n) / d(i))}
\end{aligned}
$$

Now

$$
\begin{gathered}
\prod_{i=1}^{n-1}\left[x_{1 i}^{d(i)}, x_{1 n}, x_{1 n}\right]^{-u_{i n}(d(n) / d(i))} \\
\quad=\prod_{i=1}^{n-1}\left\{\left[x_{1 i}, x_{1 n}, x_{1 n}^{d(n)}\right]^{-u_{i n}}\right.
\end{gathered}
$$

$$
\begin{aligned}
\left.\cdot\left[x_{1 n}, x_{1 i}, x_{1 i}, x_{1 n}\right]^{u_{i n}(d(n) / d(i))(} \begin{array}{c}
d(i) \\
2
\end{array}\right) \\
\left.\cdot\left[x_{1 n}, x_{1 i}, x_{1 n}, x_{1 n}\right]^{u_{i n}\left(\begin{array}{c}
d(n) \\
2
\end{array}\right)}\right\}=1, \\
\text { as } 2=d(i) \text { divides } \frac{d(n)}{d(i)} .
\end{aligned}
$$

Also it can be seen easily from (7) that

$$
\prod_{i=1}^{n-1}\left[x_{1 i}^{d(i)}, x_{1 n}, x_{1 n}\right]^{-2 w_{i n n}}=1 \text {. }
$$

Now using (38), (40), (43), (44), and (45), we get that

$$
\begin{aligned}
1= & \prod_{i=1}^{n-1}\left[x_{1 i}^{d(i)}, x_{1 n}, x_{1 n}\right]^{-u_{i n}(d(n) / d(i))} \\
& \cdot \prod_{i=1}^{n-1}\left[x_{1 i}^{d(i)}, x_{1 n}, x_{1 n}\right]^{-w_{i n n}}=g^{-1} .
\end{aligned}
$$

Hence, $g=1$.

\section{Groups of Class 3}

It has been proved that if $G$ is a finite $p$-group of class $3, p$ odd prime, then $D_{4}(G)=\{1\}$. Clearly for such a group $G, D_{5}(G)=$ $\{1\}$. Now the question arises that if $G$ is 2 group of class 3 with $D_{4}(G) \neq\{1\}$, then is it possible that $D_{5}(G)=\{1\}$ ? We will discuss some examples of 2 groups of class 3 in which $D_{4}(G) \neq$ $\{1\}$, but $D_{5}(G)=\{1\}$.

3.1. Example 1. Let $G$ be a group generated by four elements $r, a, b, c$. Set $x_{0}=y_{0}=z_{0}=r$ and $x_{i}=\left[x_{i-1}, a\right], y_{i}=\left[y_{i-1}, b\right]$, and $z_{i}=\left[z_{i-1}, c\right], i=1,2,3$. Suppose that $G$ satisfies the relations

(i) $r^{2^{7}}=1, a^{2^{6}}=y_{1}{ }^{4} z_{1}{ }^{2}, b^{2^{4}}=x_{1}{ }^{-4} z_{1}$, and $c^{2^{2}}=$ $x_{1}^{-2} y_{1}^{-1}$

(ii) $z_{2}=y_{2}{ }^{4}, y_{2}=x_{2}{ }^{4}$;

(iii) $x_{3}=y_{3}=z_{3}=1$;

(iv) $[a, b, g]=1,[b, c, g]=[a, c, g]=1 \forall g \in G$;

(v) $\left[x_{i}, b\right]=\left[x_{i}, c\right]=\left[y_{i}, a\right]=\left[y_{i}, c\right]=\left[z_{i}, a\right]=\left[z_{i}, b\right]=$ $1 \forall i \geq 1$;

(vi) $\left[x_{i}, x_{j}\right]=\left[x_{i}, y_{j}\right]=\left[x_{i}, z_{j}\right]=\left[y_{i}, y_{j}\right]=\left[y_{i}, z_{j}\right]=$ $\left[z_{i}, z_{j}\right]=1 \forall i, j \geq 0$. It has been proved in [6] that $G$ is 2 group of class 3 , and $D_{4}(G) \neq\{1\}$. we will show that $D_{5}(G)=\{1\}$.

From the above relations we conclude that

(i) $[a, b]^{2^{4}}=x_{2}{ }^{4},[b, c]^{2^{2}}=y_{2}=x_{2}{ }^{4}$, and $[a, c]^{2^{2}}=x_{2}{ }^{2}$;

(ii) $o\left(x_{1}\right)=2^{7}, o\left(x_{2}\right)=2^{6}, o\left(y_{1}\right)=2^{5}, o\left(y_{2}\right)=2^{4}, o\left(z_{1}\right)=$ $2^{3}$, and $o\left(z_{2}\right)=2^{2}$, where $o(g)$ denotes the order of an element $g$. 
Consider

$$
\begin{aligned}
& x_{11}=c, \\
& x_{12}=b, \\
& x_{13}=a, \\
& x_{14}=r ; \\
& x_{21}=[a, c], \\
& x_{22}=[b, c], \\
& x_{23}=[r, c], \\
& x_{24}=[a, b], \\
& x_{25}=[r, b], \\
& x_{26}=[r, a] ; \\
& x_{31}=[r, a, a]=x_{2} .
\end{aligned}
$$

It is easy to see that $d(1)=2^{2}, d(2)=2^{4}, d(3)=2^{6}, d(4)=2^{7}$, $e(1)=2^{2}, e(2)=2^{2}, e(3)=2^{2}, e(4)=2^{4}, e(5)=2^{4}, e(6)=2^{6}$, and $f(1)=2^{6}$. Also with the help of (1) and (2), we get that $b_{15}=-1, b_{16}=-2, b_{23}=1, b_{26}=-4, b_{33}=2$, and $b_{35}=4$.

Since $G$ is a group of class 3 , so an arbitrary element of $D_{5}(G)$ can be written as

$$
\begin{aligned}
g & =\prod_{1 \leq i<j \leq 4}\left[x_{1 i}, x_{1 j}\right]^{u_{i j} d(j)}=[c, b]^{16 u_{12}}[c, a]^{64 u_{13}} \\
& \cdot[c, r]^{128 u_{14}}[b, a]^{64 u_{23}}[b, r]^{128 u_{24}}[a, r]^{128 u_{34}} \\
= & x_{2}^{-16 u_{12}-32 u_{13}-16 u_{23}} .
\end{aligned}
$$

Equation (9) for $i=1$ and $k=6$ becomes

$$
\begin{aligned}
0 & =u_{12} b_{26}+u_{13} b_{36}+u_{14} b_{46}+v_{16} d(1)+v_{16}^{\prime} e(6) \\
& =-4 u_{12}+4 v_{16}+64 v_{16}^{\prime},
\end{aligned}
$$

which gives that

$$
-u_{12}+v_{16} \equiv 0 \quad(\bmod 4) .
$$

Also (17), for $k=6$, gives that

$$
v_{16}+2 v_{26} \equiv 0 \quad(\bmod 16) .
$$

For $k=3$ and $l=6$, (18) becomes

$$
v_{26} \equiv 0 \quad(\bmod 2) .
$$

Now, (50), (51), and (52) imply that

$$
u_{12} \equiv 0 \quad(\bmod 4) \text {. }
$$

Again, for $i=3$ and $k=6$, (9) becomes

$$
32 u_{13}+16 u_{23} \equiv 0 \quad(\bmod 64) \text {. }
$$

Since order of $x_{2}$ is 64, combining (53) and (54), we get that $g=1$.
3.2. Example 2. Let $G$ be a group generated by four elements $x_{1}, x_{2}, x_{3}, x_{4}$. Set $y_{1}=\left[x_{2}, x_{3}\right], y_{2}=\left[x_{1}, x_{2}\right], y_{3}=\left[x_{1}, x_{3}\right]$, and $y_{4}=\left[x_{1}, x_{4}\right]$. Suppose that $G$ satisfies the following relations:

$$
\begin{aligned}
{\left[x_{2}, y_{2}\right] } & =y_{1}{ }^{4}, \\
{\left[x_{3}, y_{3}\right] } & =y_{1}{ }^{-16}, \\
{\left[x_{4}, y_{4}\right] } & =y_{1}{ }^{64} ; \\
{\left[x_{1}, y_{q}\right] } & =1, \quad 1 \leq q \leq 4 ; \\
{\left[x_{2}, y_{q}\right] } & =1, \quad 1 \leq q \leq 4, q \neq 2 ; \\
{\left[x_{3}, y_{q}\right] } & =1, \quad 1 \leq q \leq 4, q \neq 3 ; \\
{\left[x_{4}, y_{q}\right] } & =1, \quad 1 \leq q \leq 4, q \neq 4 ; \\
x_{1}{ }^{k^{k+l+4}} & =y_{1}{ }^{-2^{k+l+4}} y_{2}^{2^{k+l+3}} ; \\
x_{2} 2^{2^{k+4}} & =y_{3}{ }^{2^{k}} y_{4}{ }^{-2^{k-1}} y_{1}^{3.2^{k+3}} ; \\
x_{3} 2^{2^{k+2}} & =y_{1}{ }^{-5.2^{k+1}} y_{2}^{2^{k}} y_{4}^{2^{k-2}} ; \\
x_{4}{2^{k}}^{k} & =y_{1}^{2^{k}} y_{2}^{2^{k-1}} y_{3}^{2^{k-2}} .
\end{aligned}
$$

Consider

$$
\begin{aligned}
& x_{11}=x_{4}, \\
& x_{12}=x_{3}, \\
& x_{13}=x_{2}, \\
& x_{14}=x_{1} ; \\
& x_{21}=y_{1}, \\
& x_{22}=y_{4}, \\
& x_{23}=y_{3}, \\
& x_{24}=y_{12} ; \\
& x_{31}=\left[x_{2}, y_{2}\right]=y_{1}{ }^{4} .
\end{aligned}
$$

It is easy to see that $d(1)=2^{k}, d(2)=2^{k+2}, d(3)=2^{k+4}$, $d(4)=2^{k+l+4}, e(1)=4, e(2)=2^{k}, e(3)=2^{k+2}, e(4)=2^{k+4}$, and $f(1)=2^{k+2}$. Also we have $b_{11}=2^{k}, b_{13}=2^{k-2}, b_{14}=2^{k-1}$, $b_{21}=-5.2^{k+1}, b_{22}=2^{k-2}, b_{24}=2^{k}, b_{31}=3.2^{k+3}, b_{32}=-2^{k-1}$, $b_{33}=2^{k}, b_{41}=-2^{k+l+4}$, and $b_{44}=2^{k+l+3}$. It has been proved in [7] that $G$ is a group of class 3 and $D_{4}(G) \neq 1$. We will prove that $D_{5}(G)=\{1\}$.

An arbitrary element $g$ of $D_{5}(G)$ can be written as

$$
\begin{aligned}
g & =\prod_{1 \leq i<j \leq 4}\left[x_{1 i}, x_{1 j}\right]^{u_{i j} d(j)}=\left[x_{4}, x_{3}\right]^{u_{12} 2^{k+2}} \\
& \cdot\left[x_{4}, x_{2}\right]^{u_{13} 2^{k+4}}\left[x_{4}, x_{1}\right]^{u_{14} 2^{k+l+4}}\left[x_{3}, x_{2}\right]^{u_{23} 2^{k+4}} \\
& \cdot\left[x_{3}, x_{1}\right]^{u_{24} 2^{k+l+4}}\left[x_{2}, x_{1}\right]^{u_{34} 2^{k+l+4}} \\
& =y_{1}{ }^{2^{k+4}\left(u_{12}-2 u_{13}-u_{23}+2^{l+1} u_{34}\right)} .
\end{aligned}
$$


To show that $g=1$, it is enough to show that $u_{12}-2 u_{13}-u_{23}+$ $2^{l+1} u_{34} \equiv 0(\bmod 4)$, since order of $y_{1}$ is $2^{k+6}$.

Now, for $i=3$ and $k=4$, (9) becomes

$$
\begin{aligned}
0= & u_{34} b_{44}-u_{13} \frac{d(3)}{d(1)} b_{14}-u_{23} \frac{d(3)}{d(2)} b_{24}+v_{34} d(3) \\
& +v_{34}^{\prime} e(4) \\
= & 2^{k+2}\left(2^{l+1} u_{34}-2 u_{13}-u_{23}+4 v_{34}+4 v_{34}^{\prime}\right)
\end{aligned}
$$

which implies that

$$
2^{l+1} u_{34}-2 u_{13}-u_{23} \equiv 0 \quad(\bmod 4) .
$$

Also (9) for $i=1$ and $k=2$ gives that

$$
\begin{aligned}
0 & =u_{12} b_{22}+u_{13} b_{32}+u_{14} b_{42}+v_{12} d(1)+v_{12}^{\prime} e(2) \\
& =2^{k-2} u_{12}-2^{k-1} u_{13}+2^{k} v_{12}+2^{k} v_{12}^{\prime} .
\end{aligned}
$$

That is, we have

$$
u_{12}=2 u_{13}-4 v_{12}-4 v_{12}^{\prime} .
$$

Again (9), for $i=1, k=3$, gives that

$$
u_{13} \equiv-v_{13} \quad(\bmod 4) .
$$

Now (17) for $k=3$ gives that

$$
v_{13} \equiv 0 \quad(\bmod 4) .
$$

Combining (61), (62), and (63), we get that

$$
u_{13} \equiv 0 \quad(\bmod 4) \text {, }
$$

which together with (59) gives that $g=1$.

\section{Competing Interests}

The author declares no competing interests.

\section{References}

[1] I. B. S. Passi, Group Rings and Their Augmentation Ideals, vol. 715 of Lecture Notes in Mathematics, Springer, Berlin, Germany, 1979.

[2] I. B. Passi, "Dimension subgroups," Journal of Algebra, vol. 9, pp. 152-182, 1968.

[3] E. Rips, "On the fourth integer dimension subgroup," Israel Journal of Mathematics, vol. 12, pp. 342-346, 1972.

[4] K.-I. Tahara, "The augmentation quotients of group rings and the fifth dimension subgroups," Journal of Algebra, vol. 71, no. 1, pp. 141-173, 1981.

[5] I. B. Passi and R. Mikhailov, Lower Central and Dimension Series of Groups, vol. 1952 of Lecture Notes in Mathematics, Springer, Berlin, Germany, 2009.

[6] N. Gupta, "The dimension subgroup conjecture," The Bulletin of the London Mathematical Society, vol. 22, no. 5, pp. 453-456, 1990.

[7] K.-I. Tahara, "The fourth dimension subgroups and polynomial maps II," Nagoya Mathematical Journal, vol. 69, pp. 1-7, 1978. 


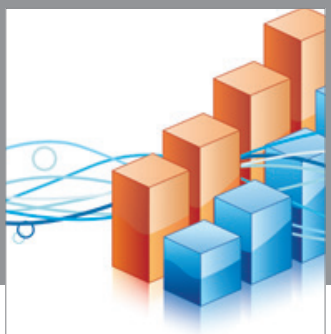

Advances in

Operations Research

vatem alat4

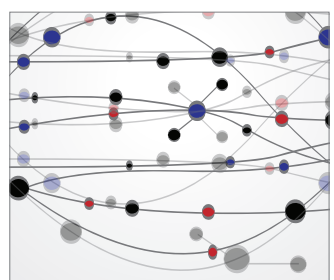

\section{The Scientific} World Journal
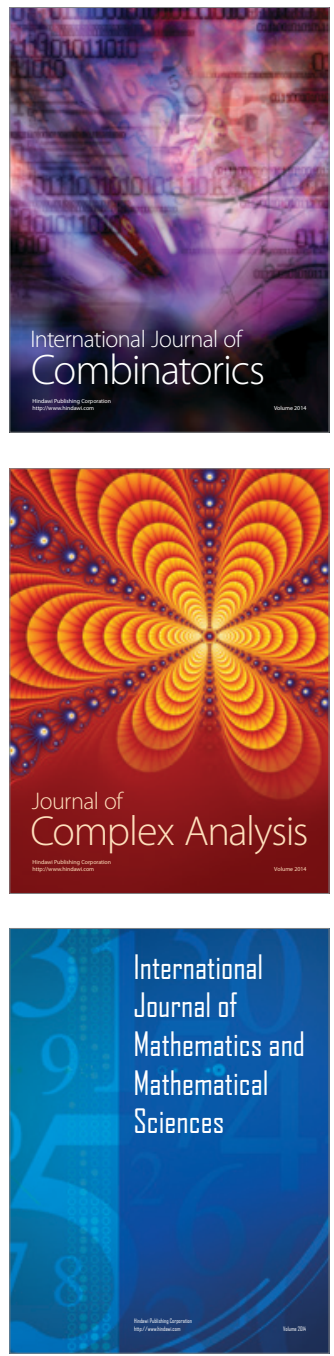
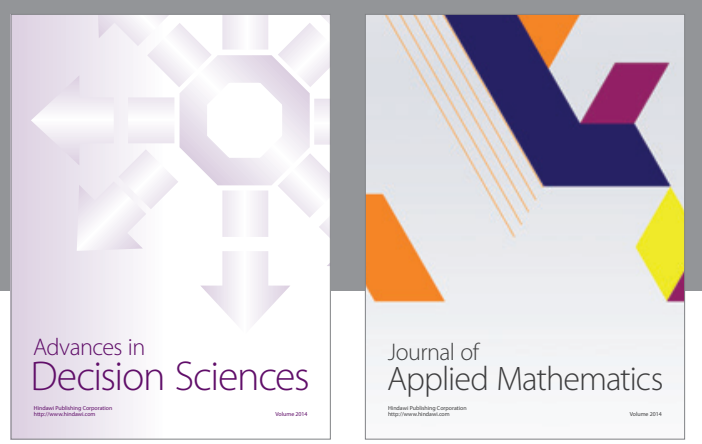

Algebra

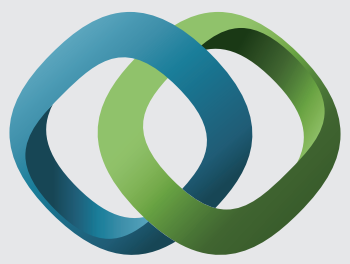

\section{Hindawi}

Submit your manuscripts at

http://www.hindawi.com
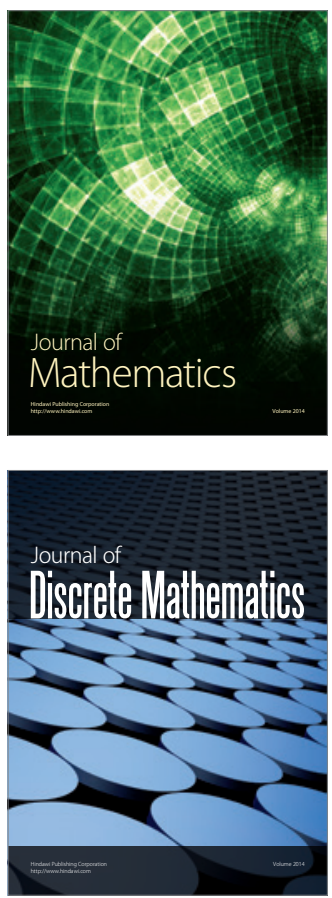

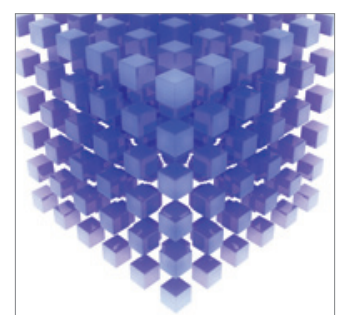

Mathematical Problems in Engineering
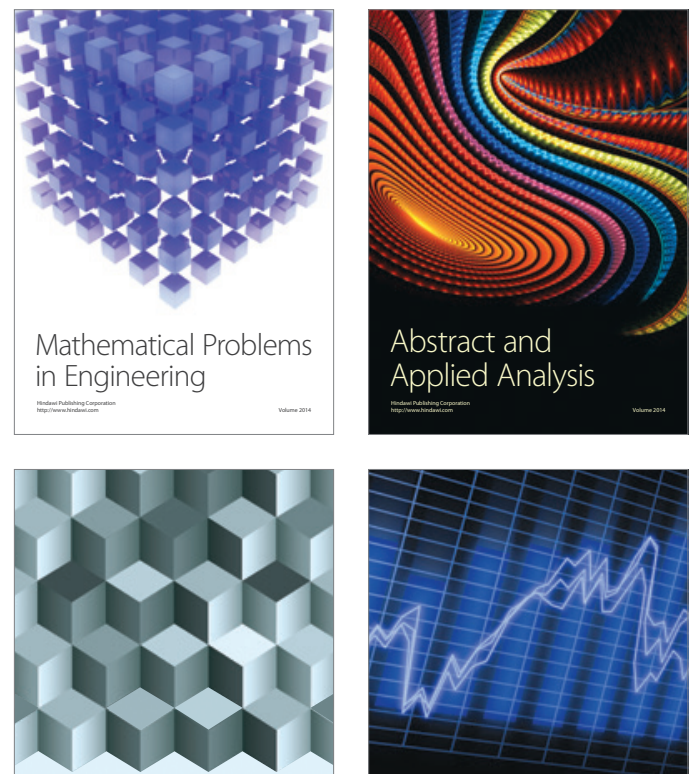

Journal of

Function Spaces

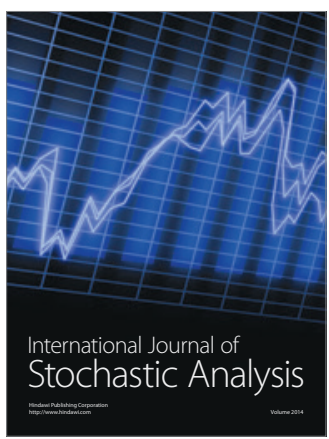

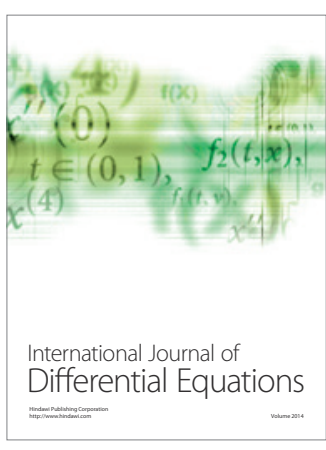
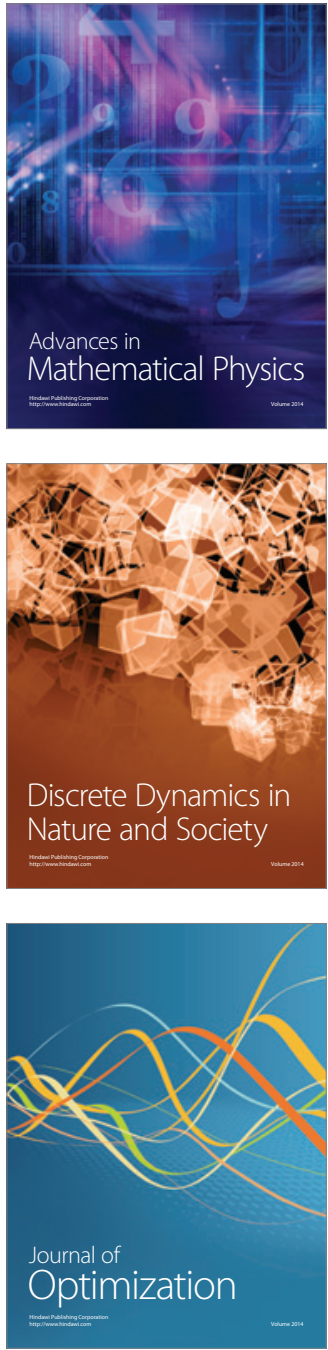